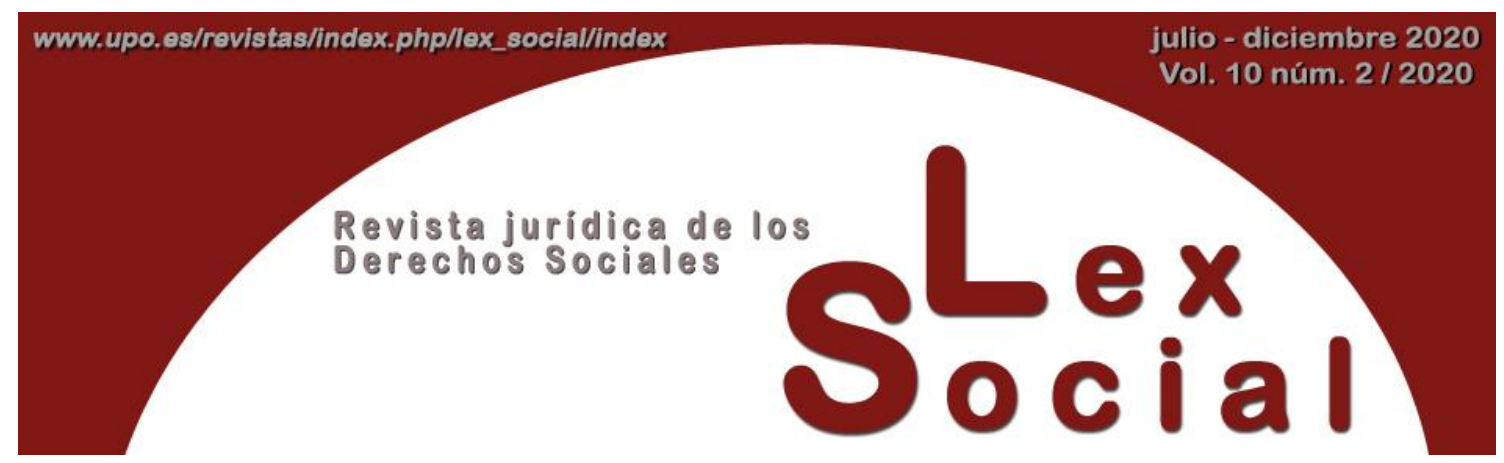

\title{
DERECHO A LA DESCONEXIÓN DIGITAL: UN ESTUDIO DE LA NEGOCIACIÓN COLECTIVA
}

\section{RIGHT TO DIGITAL DISCONNECTION: AN ANYLISIS OF THE COLLECTIVE BARGAINING AGREEMENTS}

Artículo recibido el 4 de mayo de 2020

Artículo aceptado el 8 de mayo de 2020

\author{
ÓSCAR REQUENA MONTES ${ }^{1}$ \\ Universitat Rovira $i$ Virgili
}

DOI: https://doi.org/10.46661/lexsocial.5076

\begin{abstract}
RESUMEN
En el presente artículo se estudia el derecho a la desconexión tecnológica desde una perspectiva jurídico-laboral. El objetivo es reflexionar acerca del contenido y la eficacia del mismo, considerando los antecedentes en otros países para posteriormente comparar y analizar varios textos legislativos, pronunciamientos judiciales y, especialmente, los acuerdos alcanzados a través de la negociación colectiva.
\end{abstract}

Palabras Clave: Desconexión digital, negociación colectiva, derecho al descanso, tiempo de trabajo.

\section{Abstract}

Throughout this research paper is been studied, from a Labour Law perspective, the right to digital disconnection. The aim of this work is to reflect on its content and efficacy. For this purpose, it has been considered the background information taken

\footnotetext{
${ }^{1}$ Este trabajo se inscribe en el trabajo y resultados del Proyecto DER2017-83488-C4-3-R "Los derechos fundamentales ante el cambio del trabajo subordinado en la era digital" financiado por el Ministerio de Ciencia, Innovación y Universidades, la Agencia Estatal de Investigación y FEDER.
} 
from other countries in order to compare and to analyse some legal texts, sentences and, especially, the collective bargaining agreements.

KEYWORDS: Digital disconnection, collective bargaining, right to rest, working time.

SUMARIO

\section{INTRODUCCIÓN.}

1. LA LUCHA CONTRA LA HIPERCONECTIVIDAD.

1.1. Primeros pasos.

1.2. La desconexión digital en la norma legal española.

2. ¿EN QUÉ SE CONCRETA LA DESCONEXIÓN?

2.1. Las primeras respuestas de los tribunales.

2.2. El desarrollo del derecho por parte de la negociación colectiva.

CONCLUSIONES.

BIBLIOGRAFÍA.

ANEXO. LISTADO DE CONVENIOS COLECTIVOS Y PLANES DE IGUALDAD CON

REFERENCIA EXPRESA A LA DESCONEXIÓN DIGITAL.

\section{INTRODUCCIÓN.}

El tiempo de descanso se ha comprendido tradicionalmente como aquél que acoge los tiempos de carácter personal o autodeterminables, ya vengan éstos dedicados a la familia o a la formación y a su desarrollo cultural como persona, al ocio o, simplemente, a "no hacer nada"2.

Esa constante separación entre el tiempo y el espacio de trabajo respecto del tiempo de descanso es una realidad que viene difuminándose $\mathrm{e}^{3}$ y que, especialmente las nuevas generaciones, los llamados "nativos tecnológicos", han normalizado. La confusión de los tiempos de ocio y de trabajo, a modo de totum revolutum supone, no obstante, un riesgo para su salud física y psicológica.

En efecto, la elevada implantación de los descubrimientos tecnológicos, de cuyos avances no se libra casi ningún ámbito ni tarea, conduce a las personas a verse sometidas al constante contacto con los dispositivos tecnológicos, llamados en un principio a simplificar y agilizar cualquier cometido. Sin embargo, nadie nos ha informado ni formado acerca del uso adecuado y razonable de la tecnología. En este sentido, no faltan

\footnotetext{
${ }^{2}$ LAFARGUE, Paul, El derecho a la pereza, Mandala, Madrid, 2017, p. 43.

${ }^{3}$ Sobre la liquidez de las dimensiones espaciotemporales: BAUMAN, Zygmunt, Modernidad líquida, Fondo de cultura económica, México, 2003.
} 
en la doctrina voces que recomiendan incluir la capacidad o competencia de desconexión como elemento esencial de la competencia digital ${ }^{4}$, integrando esta, además, en el currículum escolar ${ }^{5}$.

En la edad adulta, la prolongación e intensificación de la jornada de trabajo impide o dificulta gravemente al trabajador desconectar de su actividad laboral para dedicarse a descansar, ya sea estando sólo, acompañado de su familia o amigos o disfrutando de sus aficiones $^{6}$. El ser humano no es, desde luego, una máquina programada para trabajar a máxima intensidad y parar repentinamente.

\section{LA LUCHA CONTRA LA HIPERCONECTIVIDAD.}

Unas breves líneas servirán para apuntar, a grandes rasgos, el recorrido que ha seguido el derecho a la desconexión digital desde sus inicios, fuera de nuestras fronteras, hasta su inclusión en la normativa laboral española.

\subsection{Primeros pasos.}

Tal y como ha tenido oportunidad de señalar la doctrina, alemanes y franceses fueron los primeros en percatarse de los cambios sociológicos a los que se ha hecho referencia en el apartado anterior ${ }^{7}$. Fue en el año 2014, con ocasión del debate sobre la ley antiestrés en Alemania, cuando se propuso por primera vez que, salvo circunstancias excepcionales, se prohibiese al empresario contactar a ciertas horas con el trabajador ${ }^{8}$.

El germen de la investigación alemana estuvo en el aumento de las ausencias al trabajo en un cuarenta por ciento entre los años 2008 y 2011 debido a trastornos mentales, lo cual alertó al Instituto alemán de Salud y Seguridad (BAuA) ${ }^{9}$. Al mismo tiempo, el Grupo

\footnotetext{
${ }^{4}$ Véase NAVAL, Concepción et al, "Sobre la necesidad de desconectar: algunos datos y propuestas", Education in the Knowledge Society, vol. 17, n. 2, 2016, p. 84. También la Recomendación del Consejo de la Unión Europea, de 22 de mayo de 2018, relativa a las competencias clave para el aprendizaje permanente (2018/C 189/01), que ya entiende que la competencia digital no es sólo el uso "seguro y crítico" sino también "responsable" de las tecnologías digitales de aprendizaje.

${ }^{5}$ Propuesta de resolución del Parlamento Europeo, de 24 de noviembre de 2008, sobre alfabetización de los medios de comunicación en un mundo digital (2008/2129(INI)).

${ }^{6}$ Sobre los efectos positivos del control de los tiempos de trabajo: KARASEK, Robert, "Job demands, job decision latitude, and mental strain: implications for job redesign", Administrative Science Quarterly, no. 24, 1979, pp. 285-308.

${ }^{7}$ Véase GONZÁLEZ COBALEDA, Estefanía, "Riesgos psicosociales, derechos fundamentales y NTIC: una perspectiva de protección diferente”, en CEF-RTSS Comentarios y Casos Prácticos, núm. 387, 2015, pp. 25-28 y AGUILERA IZQUIERDO, R. y CRISTÓBAL RONCERO, R., "Nuevas tecnologías y tiempo de trabajo: el derecho a la desconexión tecnológica", en AA.VV., El futuro del trabajo que queremos, Vol. 2, Madrid, 2017, pp. 331-342.

${ }^{8}$ VALLECILLO GÁMEZ, María Rosa, "El derecho a la desconexión: ¿ "Novedad digital” o esnobismo del "viejo" derecho al descanso?", CEF-RTSS, núm. 408, 2017, p. 138.

9 ROTA, Anna, op. cit., p. 292, cita 1. También se ha apuntado que la causa mayor de jubilaciones anticipadas en Alemania es la salud psicosocial: MOLINA NAVARRETE, Cristóbal, "Jornada laboral y tecnologías de la info-comunicación: "Desconexión digital", garantía del derecho al descanso", Temas Laborales, núm. 138, 2017, p. 257, cita 10.
} 
Volskwagen aprobaba un acuerdo mediante el que paralizaba la circulación de correos electrónicos en las Blackberry de sus empleados durante los tiempos de no trabajo ${ }^{10}$.

Por su parte, el primer antecedente en materia de negociación colectiva hay que situarlo en Francia. Concretamente, es el artículo 17 del Acuerdo Nacional Interprofesional (ANI), de 19 de junio de 2013, el que destaca tanto las ventajas que ofrecen las TIC a las relaciones laborales como los peligros que éstas comportan, especialmente en cuanto a confusión del ámbito personal y laboral. En esta línea, se instaba a las empresas a llevar a cabo tres acciones: en primer lugar, a modo de estudio de campo, han de preocuparse de realizar un diagnóstico previo preguntando la opinión de sus trabajadores en torno a las TIC; en segundo lugar, de acuerdo con ese feedback, han de ofrecerles la posibilidad de realizar los cursos de formación y sensibilización oportunos; c) por último, las empresas deben implantar de forma efectiva ciertos tiempos de desconexión como medio para conciliar la vida profesional y personal.

Prontamente, algunas grandes compañías de nuestro país vecino acogieron en sus acuerdos colectivos dicha materia, aunque la ambigüedad y la generosidad en cuanto a la inclusión de excepciones a su cumplimiento les reportó un buen número de críticas ${ }^{11}$. Excepciones que, por lo demás, se han venido reproduciendo en los textos convencionales españoles, como tendremos ocasión de ver en las próximas páginas.

El Informe METTLING se publicó poco después, en el mes de marzo de 2015, a petición del Ministerio de Trabajo francés, enunciando el derecho a la desconexión digital como un verdadero derecho-deber de corresponsabilidad, es decir, reconociéndolo y requiriéndoselo a todas las partes firmantes del contrato porque su efectividad "depende tanto de la educación a nivel individual como de la regulación a nivel de la empresa" 12 .

Seguidamente, fue la Loi El Khomri ${ }^{13}$ la que vio en el derecho a la desconexión una oportunidad para compensar los fuertes recortes en derechos laborales que supuso la reforma laboral francesa de 2016, incorporándolo como pura facultad del trabajador, si bien su definición y delimitación reducía sus efectos de manera considerable ${ }^{14}$. En fin, cabe avanzar que esta regulación ha supuesto una "guía de ruta" para otros legisladores nacionales como el italiano, el belga o, como ahora observaremos, también para el español.

\footnotetext{
${ }^{10}$ Grupo Volkswagen, en 2011, enuncia esta especie de "toque de queda digital" consistente en el bloqueo de acceso a comunicaciones corporativas en el teléfono móvil desde las 18:15 hasta las 07:00 horas.

${ }^{11}$ Véase el artículo 20 del Acuerdo del Grupo Arèva, firmado el 31 de mayo de 2012. Asimismo, los convenios colectivos de empresas como Thalès (2014) y Orange, Michelin o Natixis (2016).

${ }^{12}$ METTLING, Bruno, "Informe Mettling, para adaptar el trabajo a la transformación digital", Ministerio de Trabajo, Migraciones y Seguridad Social: Actualidad Internacional Sociolaboral, núm. 194, 2015, p. 136.

${ }^{13}$ Aunque se conoce así por la ministra que la impulsó, se trata de la Loi Travail $\mathrm{N}^{\circ} 2016-1088$, de 8 de agosto de 2016.

${ }^{14}$ Entre los estudios publicados en España, uno de los más detallados es el elaborado por ALEMÁN PÁEZ, Francisco, "El derecho a la desconexión digital. Una aproximación conceptual, crítica y contextualizadora al hilo de la "Loi Travail No 2016-1088”", Trabajo y Derecho, núm. 30, 2017, pp. 12-33.
} 


\subsection{La desconexión digital en la norma legal española}

Una vez contextualizado el origen de la regulación de la desconexión digital en otros ordenamientos jurídicos y antes de pasar a examinar los primeros desarrollos en sede convencional, resulta necesario conocer la regulación específica de este derecho tan singular en el ordenamiento jurídico español.

La primera cuestión a discutir sería la elección del momento y lugar en el que se ha decidido regular tal aspecto. No en vano, por sus implicaciones podría haber sido incluido en otros apartados de la norma laboral básica como expresión del derecho al descanso (arts. 34 y 37 ET); o también, por su potencialidad como generadora de riesgos laborales, sería incorporable a los artículos 14 y 19 de la LPRL ${ }^{15}$.

Sensu contrario, atendiendo al vínculo tecnología-intimidad que habita en tal derecho, el legislador español ha considerado oportuno mencionarlo en un nuevo artículo 20 bis del Estatuto de los Trabajadores, puesto que la auténtica regulación y desarrollo se contiene en el artículo 88 de la Ley Orgánica 3/2018, de 5 de diciembre, de Protección de Datos y garantía de los derechos digitales (en adelante, LOPDGDD).

No cabe duda de que el derecho a la desconexión digital queda recubierto y se sostiene sobre la base de varios derechos fundamentales, entre ellos el derecho a la intimidad. En consecuencia, podría afirmarse que el derecho a la desconexión digital es realmente una expresión singular del derecho a la intimidad -como también de otros derechos básicos igualmente reconocidos al trabajador en la relación laboral en el propio ET- en una dimensión específica caracterizada por la tecnología.

En cambio, sorprende la técnica legislativa empleada -mediante la proclamación del derecho en el artículo 20 bis ET $^{16}$. En efecto, el derecho de desconexión digital queda vinculado constantemente al derecho fundamental a la intimidad personal y laboral, pero la redacción del precepto lo deslinda del derecho "a la intimidad en relación con el entorno digital", pareciendo, de este modo, querer ponerse en tela de juicio el carácter "fundamental" del derecho a la desconexión.

Con independencia de lo anterior y yendo al quid de la cuestión, el artículo 18 de la LOPDGDD clasifica en tres apartados el desarrollo legal del derecho a la desconexión digital. Primeramente, el precepto declara solemnemente, pero sin apenas detalle, el derecho a la desconexión digital, al tiempo que selecciona dentro de su ámbito subjetivo de aplicación a los “trabajadores y los empleados públicos”, sin precisar el régimen en el

\footnotetext{
${ }^{15}$ La doctrina francesa ha comprendido que el objeto de análisis es el derecho a la conciliación, pero que detrás de esa cuestión está la del derecho a la salud: MATHIEU-GÉNIAUT, Chantal et al, "Le droit à la déconnexion: une chimère?", Revue de droit du travail, no. 10, 2016, p. 592. Coincido, en todo caso, en lo inoportuno que sería limitarla a su aspecto conciliador de la vida laboral y personal, pues este asunto no es, a mi modo de ver, el más relevante, ni el más íntimamente relacionado con el derecho que estamos examinando.

${ }^{16}$ Como bien ha indicado el profesor BAYLOS GRAU, ello debería haberse efectuado en el artículo 4 ET. Vid. "La garantía de los derechos digitales en el trabajo. El proyecto de Ley ya aprobado en el Congreso. Un texto decepcionante", en su blog Según Antonio Baylos..., disponible en https://baylos.blogspot.com/2018/11/la-garantia-de-los-derechos-digitales.html (última consulta: 29.10.2019).
} 
que éstos prestan servicios, por lo que, desde mi punto de vista, merece ser comprendido de manera amplia.

Asimismo, el legislador enuncia vagamente las finalidades jurídico-prácticas del derecho a la desconexión, así como los otros derechos generales que, por su íntima conexión con aquél, deben quedar reforzados a través del mismo. En el primer apartado, se refiere al derecho al descanso y al derecho a la intimidad personal y familiar; en estrecha relación con éste, se cita en el apartado segundo el derecho a la conciliación de la actividad laboral con la vida personal y familiar; en último lugar, el apartado tercero insiste sobre el derecho a la salud, esta vez para animar a evaluar y tratar, dentro de la política interna de la empresa y mediante acciones de formación y sensibilización, el riesgo de "fatiga informática" como consecuencia de un uso indebido o abuso de las herramientas tecnológicas.

Sin lugar a dudas, el punto más criticable de la regulación legal del derecho lo encontramos en su eficacia jurídica. No solo porque carece de una mínima definición del derecho en sí mismo, sino por no haberse observado garantías legales que permitieran presumir un cambio radical de comportamiento por parte de los agentes sociales y de las partes individuales del contrato, como seguidamente comprobaremos. No obstante, el modo de implementación del derecho, queda absolutamente relegado a lo que las partes convengan en la negociación colectiva -con la mera recomendación de que la regulación se adapte a la naturaleza y objeto de la relación laboral ${ }^{17}$.

Además, ninguna de las partes se va a sentir obligada a sentarse a negociar, ya que el mismo no forma parte del contenido obligatorio de los convenios colectivos (art. 85.3 ET). Tanto es así que el propio legislador "se pone la venda antes de la herida" al subdelegar explícitamente en "lo acordado entre la empresa y los representantes legales de los trabajadores"18. Las posibilidades de que este asunto se resuelva por acuerdo entre las partes se reducen si tenemos en cuenta, además, que la gran mayoría de empresas españolas carecen de representantes legales de los trabajadores.

Pues bien, siendo reducido el contenido del artículo 88 de la LOPDPGDD, no por ello cabe negar que pueda tener alguna utilidad o efecto. A mi modo de ver, lejos de considerarlo un derecho líquido, el mero hecho de haberse reconocido el derecho a la desconexión digital, de manera expresa y en congruencia con el trabajo decente, ha de reforzar la tutela preventiva del derecho de los trabajadores a la conciliación laboral y personal y del derecho-deber del trabajador y del empresario al descanso y a la salud -en

\footnotetext{
${ }^{17}$ A tenor de lo previsto en el art. 88.3 in fine, parece estar refiriéndose, de manera especial, a los supuestos de teletrabajo, en el domicilio o "a distancia", sin perjuicio de los debidos ajustes que requieran otras actividades o modos de prestar servicios. Recuérdese que la STSJ de Castilla y León, Sala de lo Social, de 3 de febrero de 2016, sugiere que las empresas que hacen uso de las TIC o del teletrabajo deberían gestionar el tiempo estableciendo, por ejemplo, procedimientos de conexión digital o registros de jornada. El convenio colectivo del grupo Marítima Dávila, SA (BOE de 11.11.2018) ya fomenta el teletrabajo y se acuerda del derecho a la desconexión digital, pero de modo tan superficial que ni siquiera será tenido en cuenta en el estudio de la negociación colectiva con el que finaliza este trabajo.

${ }^{18}$ En el panorama europeo encontramos ejemplos donde los agentes sociales sí se han interesado por regular la cuestión (Francia) y otros donde no (Italia). Cfr. ROTA, Anna, op. cit., p. 303.
} 
esta ocasión, vinculado a las tecnologías y a modo de obligación de resultados del empresario $^{19}$.

En efecto, en una primera aproximación podría decirse que el derecho a la desconexión digital no es, por sí mismo, un derecho fundamental, ya que, al menos formalmente, no parece serlo, puesto que en ningún sitio aparece declarado como tal y, aun apareciendo regulado en una Ley Orgánica, el concreto artículo que lo regula -el artículo 88- carece de ese carácter $^{20}$. En cambio, el derecho a la desconexión digital no deja de ser una particular expresión del derecho fundamental al descanso ${ }^{21}$, que además se reconoce al abrigo de, como mínimo, otros cuatro derechos, de los cuales varios se declaran directamente como fundamentales en la propia Constitución Española. Estos son: el derecho a la salud ${ }^{22}$, el derecho a la vida y a la integridad física y moral ${ }^{23}$, el derecho a la conciliación de la vida laboral, familiar y personal ${ }^{24}$, el derecho a la intimidad personal ${ }^{25}$ $\mathrm{y}$, como derecho fundamental laboral inespecífico ${ }^{26}$ que le es reconocido a todo trabajador, el debido respeto a la dignidad.

En definitiva, son muchos los argumentos que obligan al empresario a "tomar cartas en el asunto" y elaborar una política interna real en contra de la hiperconectividad de sus trabajadores. Este deber ha quedado positivizado en la nueva normativa, que remite al acuerdo mediante negociación colectiva o con los representantes de los trabajadores y, en su defecto, a la actuación directa del empresario. Dicha "política interna" ha de contener, al menos, dos aspectos: por un lado, la selección y definición del modo o modos en los que se va a implementar; por otro lado, ha de definir las acciones formativas y de sensibilización dirigidas a sus trabajadores al fin de que aprendan a usar razonablemente los dispositivos electrónicos. Esta segunda vertiente sería reflejo del derecho-obligación

\footnotetext{
${ }^{19}$ A favor, PURCALLA BONILLA, Miguel Ángel, "Control tecnológico de la prestación laboral y derecho a la desconexión: claves teóricas y práctica”, Anuario IET de trabajo y relaciones laborales, Vol. 5, 2018, p. 109.

20 Tiene naturaleza de ley ordinaria, en virtud de lo dispuesto en la Disposición final primera de la LOPDGDD.

${ }^{21}$ Entre muchas otras normas, véase el artículo 31.2 de la Carta de los Derechos Fundamentales de la Unión Europea y el Convenio de la OIT núm. 132.

${ }^{22}$ Entre otros preceptos, véase el artículo 25 de la Declaración Universal de los Derechos Humanos y el artículo 12 del Pacto Internacional de Derechos Económicos, Sociales y Culturales.

${ }^{23}$ Artículo 15 de la Constitución Española. En la relación de trabajo propiamente se reconoce en virtud del artículo 4.2.d) ET.

${ }^{24}$ Artículo 44 de la Ley Orgánica 3/2007, de 22 de marzo, para la igualdad efectiva de mujeres y hombres, y artículo $34.8 \mathrm{ET}$, entre otros.

${ }_{25}$ Artículo 18 de la Constitución Española. Véanse asimismo los artículos 4.2.e) y 20.bis del ET, que se citarán a continuación.

${ }^{26}$ PALOMEQUE LÓPEZ, Manuel Carlos, Los derechos laborales en la Constitución Española, Centro de Estudios Constitucionales, Madrid, 1991, pp. 31-34 y 79. Véanse los artículos 10.1 CE y 4.2.e) ET.
} 
del trabajador y del empresario a la formación en materia preventiva ${ }^{27}$ o para la adaptación de aquél a las modificaciones operadas en el puesto de trabajo ${ }^{28}$.

\section{2. ¿EN QUÉ SE CONCRETA LA DESCONEXIÓN?}

De forma intencionada o no, lo que ha quedado claro es que la legislación española no aporta ninguna definición legal acerca de lo que significa la desconexión digital. Sin embargo, del término "desconectar" encontramos hasta cinco acepciones en el Diccionario de la Real Academia de la Lengua Española. Descartando aquellas que no parecen estar relacionadas con el ámbito en el que nos ubicamos, desconectar sería simple y llanamente "interrumpir la conexión entre dos o más cosas", lo que daría lugar a "dejar de tener relación, comunicación, enlace", de tal modo que, en el contexto tecnológico, llegaríamos a "interrumpir el enlace entre aparatos, o entre aparatos y personas, para que cese el flujo existente entre ellos" ${ }^{29}$. Siendo esto así, procede rescatar cuáles han sido los pronunciamientos judiciales y convencionales hasta el momento, con la finalidad de encontrar algo más de luz en la definición del derecho.

\subsection{Las primeras respuestas de los tribunales.}

Al respecto, puede traerse a colación la sentencia de la Sala de lo Social del Tribunal Superior de Justicia de Galicia ${ }^{30}$ que analiza el supuesto de una empresa que entrega a sus diez trabajadores (transportistas) un teléfono móvil y se comunica con ellos mediante un grupo de Whatsapp integrado por todos ellos y creado por aquélla. No aclara la sentencia si cuando la empresa se comunica con los trabajadores individual y conjuntamente "a través del mismo" hace referencia al grupo de Whatsapp (comunicación colectiva) o al teléfono móvil (a modo de comunicación individual). A mi parecer, si el trabajador pudiese probar haber sido notificado con cierta regularidad de algún asunto relativo a su actividad laboral, de carácter urgente o "escondido" entre multitud de mensajes del día anterior, por medio del grupo de Whatsapp y fuera de su jornada laboral, se estaría vulnerando claramente su derecho a la desconexión digital como expresión del derecho al descanso y a la intimidad y/o en su vertiente preventiva del riesgo psicosocial de estrés por sobrecarga de trabajo. Cabe aclarar, no obstante, que no parece ser éste el caso juzgado por el tribunal gallego, que se remite al juzgado de instancia y a su declaración

\footnotetext{
${ }^{27}$ Arts. 19.4 ET y 19 LPRL. Se trataría, pues, de una causa adicional a las ya previstas en dichos preceptos (inicio de la relación laboral, cambios en las funciones, introducción de nuevas tecnologías y cambios en los equipos de trabajo) y el tiempo dedicado a la formación y concienciación se sometería al régimen jurídico previsto en el artículo 19.2 LPRL.

${ }_{28}^{28}$ Arts. 23.1.d) y 52.b) ET. Sobre el estado actual de la norma, me permito remitir a REQUENA MONTES, Óscar, Los derechos individuales de formación en el artículo 23 del Estatuto de los Trabajadores, Tirant lo Blanch, Valencia, 2019, pp. 330-390.

29 Diccionario de la Real Academia de la Lengua Española, Desconectar, disponible en https://dle.rae.es/srv/search?m=30\&w=desconectar (última consulta: 29.10.2019)

${ }^{30}$ STSJ de Galicia, Sala de lo Social, de 18 de diciembre (Rec. 2852/2018).
} 
como hecho no probado el que la empresa contactase con el trabajador durante el periodo de descanso diario o semanal.

Efectivamente, lo concluido en el párrafo anterior podría justificarse por medio de otra resolución que ya ha sido debidamente examinada por la doctrina más autorizada ${ }^{31}$. La sentencia proviene concretamente de la Sala de lo Social del Tribunal Superior de Justicia de Cataluña ${ }^{32}$ y versa sobre un "acelerómetro" instalado en el teléfono móvil que la empresa proporciona a los trabajadores del área de mantenimiento, el cual se complementa con un GPS también incorporado en el dispositivo. Aunque la empresa alega que el mencionado acelerómetro sirve para detectar el movimiento del trabajador $\mathrm{y}$, así, conocer rápidamente si ha habido un accidente, queda probado por la Inspección de Trabajo que la función del mencionado acelerómetro no es la de actuar como equipo de protección individual (EPI) sino la de controlar la actividad del empleado. Lo más interesante, por cuanto al tema que aquí se está estudiando, es que: por un lado, el acelerómetro no se puede desconectar si no es mediante una aplicación de la que no tienen conocimiento los trabajadores ( $\mathrm{FJ} 9^{\circ}$ ); por otro lado, el trabajador no sólo debe llevar el acelerómetro encima -no en la caja de herramientas- durante la jornada laboral, sino que también debe llevárselo a su domicilio y encargarse de cargar la batería para que siempre esté en grado óptimo para su funcionamiento (FJ $\left.10^{\circ}\right)$.

Esto conlleva, citando literalmente la resolución judicial, una "lógica previsión y preocupación de los trabajadores [...] y la intromisión en lo que es la vida privada y familiar" (FJ $10^{\circ}$ ). No obstante, continuando con la cita, "lleva consigo una situación de riesgo psicosocial pues la circunstancia de que utilice la empresa un aparato de última tecnología para controlar el trabajo no puede tener la consecuencia de que fuera de la jornada laboral tengan incluso que en su domicilio familiar en los que es la esfera personal y privada del trabajador haya de continuar en una situación in vigilando del citado dispositivo" (FJ 12 ${ }^{\circ}$ sic., que repite de nuevo en el siguiente fundamento jurídico aclarando que ese riesgo es el de estrés). Desde luego, este supuesto es otro ejemplo más de cómo los instrumentos tecnológicos afectan directamente a la esfera más íntima del trabajador $^{33}$, a su capacidad de descanso y, en definitiva, a su salud mental.

Por lo demás, la litigiosidad en torno al derecho a la desconexión digital es, de momento, escasa. En aquellos conflictos donde se ha alegado la vulneración de tal derecho, se ha rechazado entrar en el fondo del nuevo precepto jurídico por razones tales como la producción de los hechos con antelación a su entrada en vigor ${ }^{34}$ o errores procesales tan

\footnotetext{
${ }^{31}$ GONZÁLEZ COBALEDA, E., op. cit., pp. 36-37. y MOLINA NAVARRETE, Cristóbal, op. cit., pp. 276-278.

${ }^{32}$ STSJ de Cataluña, Sala de lo Social, de 23 de mayo de 2013 (Rec. 6212/2012). La resolución examina el acelerómetro como medida de control empresarial, pero interesa su dimensión como factor de riesgo psicosocial.

33 Al respecto, ciertos convenios colectivos se esfuerzan ya en apuntar que la empresa carece de herramientas tecnológicas de escucha (25, CC de la empresa Centro Farmacéutico S.L.).

${ }^{34}$ FJ 4 de la STSJ de Galicia, Sala de lo Social, de 17 de octubre de 2019 (Rec. 3241/2019).
} 
burdos como la no citación del artículo hipotéticamente infringido, añadiéndose que, a juicio del tribunal, tampoco se producen los hechos denunciados ${ }^{35}$.

\subsection{El desarrollo del derecho por parte de la negociación colectiva.}

Entre los primeros ejemplos de regulación de la desconexión digital en la negociación colectiva destaca, por lo que respecta a España, el convenio colectivo de AXA Seguros (1), que se convirtió en viral precisamente por preverlo en su artículo catorce. Prescindiendo por el momento del examen de su contenido, baste indicar que una parte de la doctrina iuslaboralista ha calificado esta maniobra como puro "marketing de convenio", similar a algunas medidas de responsabilidad social corporativa que, a menudo, no añaden ningún derecho ${ }^{36}$.

Cabe anotar, asimismo, que a lo largo del año 2019 ya se han publicado excelentes estudios que han recogido algunos de los más principales convenios colectivos que han regulado esta materia ${ }^{37}$. Sin prescindir de las debidas consultas y comparaciones con tales trabajos, y avanzando que la muestra es distinta a las manejadas en aquellas investigaciones, a continuación se va a proceder a realizar un particular análisis, en su conjunto, de numerosos pactos colectivos. Si bien se ha querido añadir algunos acuerdos empresariales no publicados oficialmente, la mayor parte de ellos han sido encontrados, hasta la fecha en la que se cierra este trabajo -30 de abril de 2020-, a través del buscador previsto en la página Web de la Comisión Consultiva Nacional de Convenios Colectivos. Todos ellos tienen como patrón común la incorporación de al menos una mención explícita a la "desconexión digital” y, por esa razón, aparecen listados en el Anexo del presente trabajo por orden cronológico.

Pues bien, el modo en el que afrontar el desarrollo convencional del derecho es muy dispar entre unos y otros. Así pues, mientras algunos documentos han ofrecido un mayor desarrollo y, por una u otra razón, destacan positivamente $(14,17,25,28,34,37,42,44$, 52,54 y 58), algunos convenios colectivos simplemente nombran el derecho a modo de recordatorio $(12,31,33,36,39$ y 55), ya sea de forma solemne y breve $(13,19,27,35)$ o bien con excesiva retórica (40), o incluso haciendo uso de un registro tendente a lo

\footnotetext{
${ }^{35}$ FJ $^{\circ}$ de la STSJ de Galicia, Sala de lo Social, de 18 de diciembre de 2018 (Rec. 2852/2018).

${ }^{36}$ Con estos términos se expresó, por ejemplo, RODRÍGUEZ-PIÑERO ROYO, Miguel, en el turno de preguntas de la mesa sobre "Desconexión digital", del Congreso sobre Automatización, vigilancia y control de trabajadores y derechos fundamentales, Valencia, 12 de septiembre de 2019. Disponible en https://www.youtube.com/watch?time continue=89\&v=nF1QNYyPe3c (última consulta: 29.10.2019). Véase también IGARTUA MIRÓ, María Teresa, "El derecho a la desconexión digital en la Ley Orgánica 3/2018, de 5 de diciembre, de protección de datos personales y garantía de los derechos digitales: más forma que fondo en la configuración del derecho", CEF-RTSS, núm. 432, 2019, pp. 61-87.

${ }^{37}$ Entre los más recientes y centrados en la cuestión convencional: BARRIOS BAUDOR, Guillermo L., "El derecho a la desconexión digital en el ámbito laboral español: primeras aproximaciones", Aranzadi Doctrinal, núm. 1, 2019; MIÑARRO YANINI, Margarita, "La desconexión digital en la práctica negocial: más forma que fondo en la configuración del derecho", CEF-RTSS, núm. 440, 2019, pp. 5-18 y; TERRADILLOS ORMAETXEA, Miren Edurne, "El derecho a la desconexión digital en la ley y en la incipiente negociación colectiva española: la importancia de su regulación jurídica", Lan Harremanak, 2019, 42, In press, pp. 1-32.
} 
emocional, más que a lo riguroso y efectivo (17 y 28). Algunos otros se limitan a copiar o reproducir extractos de la norma legal $(15,16,57$ y 68$)$ reiterando -y postergando- la necesidad de llevar a cabo una política interna o "protocolo" (43, 60 y 64), si bien de manera excepcional puede encontrarse una suerte de protocolo o pautas sobre el modo en el que se debe implementar la política interna, donde destaca la "elaboración de un diagnóstico con objeto de que se identifiquen los supuestos en que se produce una interrupción del descanso del personal, distinguiendo en función de su tipología (recurrente o esporádico, puntual o de larga duración, conlleve una acción especial o no, medio tecnológico exigido para su resolución, etc.) y de la tipología de personal de que se trate, en particular, a fin de atender posibles situaciones asociadas al trabajo a distancia o teletrabajo, así como de realización total o parcial del trabajo en el domicilio del empleado vinculado al uso con fines laborales de herramientas tecnológicas. Considerar e identificar las necesidades específicas de la actividad para asegurar que se mantenga un servicio adecuado en todo caso, conciliando esta exigencia con el ejercicio del derecho a la desconexión" (44, 45, 46 y 48, todos ellos relativos a las instituciones ligadas al deporte -fútbol- profesional).

Por otro lado, es muy común que los preceptos dedicados al tema de la desconexión tecnológica comiencen con la frase testimonial por la que se anuncia que las partes "coinciden en la necesidad de impulsar el derecho" (1, 20, 21, 50, 62 y 69), muchas veces acompañada de una introducción/justificación sociológica de la materia $(1,22,24,37,44$, 45 y 46) o precedida de unas reglas sobre el uso adecuado y con fines profesionales de los dispositivos puestos a disposición de la empresa y los de carácter personal (25).

En cuanto a los derechos que expresamente se dice proteger a través del derecho a la desconexión digital, varios de ellos se acuerdan de todos o casi todos los derechos involucrados o afectados por la hiperconectividad tecnológica (67), mientras que otros lo asocian de forma exclusiva o muy destacada al derecho al descanso (17 y 28) o a la efectiva conciliación de la vida personal, laboral y familiar $(3,7,17,18,28,56,60$ y 66). En torno a esta dimensión, no faltan los pactos colectivos que declaran como "objetivo concreto" el hecho de "estudiar medidas que faciliten la conciliación también de los grupos de mandos y equipo directivo, tales como la desconexión digital, rango horario de las reuniones dentro y fuera de la empresa" (9 y 10).

Por su parte, el punto 8 de la "Revisión del acuerdo intersectorial de Navarra sobre Relaciones Laborales" (14) incide exclusivamente en la vertiente preventiva, haciendo especial hincapié en la formación para la gestión del tiempo como método de prevención ante los riesgos psicosociales que plantea la conectividad permanente. Ante ello, se insta a: "contemplar en los convenios la necesidad de implantar en las empresas protocolos de intervención y prevención de adicciones consensuadas con la RLT; impulsar en las empresas la realización de evaluaciones de riesgos teniendo en cuenta el género, especialmente en las situaciones relacionadas con la reproducción; dotar a los delegados de prevención de las herramientas necesarias para el desarrollo de su actividad; fomentar la coordinación entre los responsables de prevención y los técnicos de medioambiente de las empresas en aquellas cuestiones que confluyan aspectos 
preventivos y medioambientales e; impulsar la formación continua a trabajadores y delegados en materia preventiva".

Con todo, en muchos de ellos el derecho tiende a reducirse a la facultad de no responder a los mails o mensajes profesionales fuera de su jornada de trabajo $(1,11,23,29,47,50$, $59^{38}$ ), así como a llamadas profesionales $(7,65$ y 66) o, en fin, a medios tecnológicos como teléfonos y tablets con conexión de datos y ordenadores portátiles (3). Para evitar posibles confusiones o vacíos legales, algunas normas convencionales se refieren a "cualquier tecnología de la comunicación (teléfono, canales de mensajería, correos electrónicos, video-llamadas, y cualquier otro medio implantado en la empresa o al que estén acostumbrados los/as trabajadores/as)" (17, 28 y 61) o al "conjunto de las herramientas digitales nómadas" (44, 45, 46 y 48). Por lo que respecta a lo que deba entenderse por "fuera de la jornada de trabajo", ciertos pactos precisan que el tiempo libre abarca "vacaciones, días de asuntos propios, libranzas, descanso diario y semanal, permisos, incapacidades o excedencias", haciendo extensivo el derecho al "personal fuera de Convenio" (42, 54, 37 y 65).

Aún siguiendo con la reducción del derecho a la libertad para no atender a comunicaciones una vez superada la jornada laboral, algunos textos se detienen a concretarlo, indicando que la desconexión se fomentará siguiendo las siguientes condiciones o instrucciones: "No se enviarán correos electrónicos, hora y media antes ni hora y media después de la entrada y salida al puesto de trabajo, ni durante los fines de semana o vacaciones; Se recomienda la opción de "retrasar entrega" y establecerla en las horas indicadas; En caso de urgencia, se priorizará la llamada de teléfono sobre el correo electrónico. Si no fuera posible, se indicará en el propio correo electrónico el carácter de "importante" o "urgente" del mismo; Se preconiza la no conexión durante las vacaciones, periodos de suspensión, permiso retribuido o similar, así como los periodos de baja de IT común o profesional; Una vez finalizada la jornada laboral se reconoce el derecho de las personas que trabajan a no responder al teléfono, los correos electrónicos o mensajes profesionales de cualquier otro tipo, fuera de su horario laboral" (52).

En la misma línea, los convenios colectivos de empresas vinculadas a Telefónica resaltan la responsabilidad de los trabajadores y, en especial, de los coordinadores de equipo (posición referente), para cumplir con el derecho a la desconexión digital. En este sentido, "los superiores jerárquicos se abstendrán de requerir respuesta en las comunicaciones enviadas a las personas trabajadoras fuera de horario de trabajo o próximo a su finalización, siempre que pudieran suponer para los destinatarios de las mismas la realización de un trabajo efectivo que previsiblemente pueda prolongarse e invadir su tiempo de descanso. Por ello, las personas destinarias de la comunicación tendrán derecho a no responder a la misiva hasta el inicio de la siguiente jornada laboral" (42 y

\footnotetext{
${ }^{38} \mathrm{Y}$, con una más que discutible redacción, el 4, de la empresa Cicle de l'Aigua, cuyo compromiso se limita a que "procurará que no sea necesaria la respuesta".
} 
$54)^{39}$. A modo de inciso, aunque puede transcender lo digital y tecnológico, también resulta interesante el compromiso a que "la convocatoria de reuniones de trabajo, tanto a nivel interno como las que se lleven a cabo con clientes, así como la formación obligatoria, se reali(ce) teniendo en cuenta el tiempo aproximado de duración y, preferiblemente, no se ext(iendan) hasta más tarde de la finalización de la jornada ordinaria de trabajo" (42 y 54, así como también 34, 37 y 38).

Entre todos los documentos seleccionados, son dignos de elogio aquéllos que apuestan fuertemente por la eficacia del derecho y no sólo subrayan la posibilidad de no responder a los correos electrónicos, sino también la obligación (“compromiso") de las empresas de "no enviar a las personas trabajadoras de las mismas, mails o mensajes profesionales fuera del horario de trabajo" (62 y 64, y también el loable Acuerdo del BBVA -34-, aunque finalmente parece permitirlo, e, inspirado en él, el reciente Acuerdo de Caixabank -58-). Expresión convencional que venía siendo reclamada de manera insistente por la doctrina científica ${ }^{40}$.

En suma, también deben ser entendidas de forma positiva aquellas medidas que tratan de concienciar al trabajador requiriéndole "programar una respuesta automática en la que, indicando su situación y las fechas en las que no estará disponible, designe el correo de contacto para la reasignación del servicio" (37), así como "las fechas de duración" de la ausencia (42 y 54), puesto que los correos electrónicos pueden provenir de clientes que desconozcan su situación laboral.

Interpretación distinta merece, en mi opinión, el III convenio colectivo de Cash Converters SL y Sociedades Vinculadas (26), que siendo una copia prácticamente literal del convenio colectivo de Axa (1) y tras reconocer el derecho a no responder fuera de la jornada de trabajo, añade "informando de ello a la estructura de las empresas". Es decir, además de que su valor añadido tiende a nulo, parece obligar a los trabajadores a informar expresamente a la estructura de la empresa cuando no quieran responder a e-mails fuera de su jornada de trabajo ${ }^{41}$. Lamentablemente, en la misma dirección reduccionista del derecho se pronuncian otros convenios, indicando que las horas de desconexión fuera de la jornada serán objeto de negociación (21) o negándoles rotundamente tal derecho a las personas del comité de dirección, a los directores de negocio y a los gerentes cuyas funciones exigen plena disponibilidad (71). Tales precisiones vacían total o parcialmente el derecho a la desconexión tecnológica y, por ello, deberían ser consideradas nulas.

Por el contrario, el derecho a no responder a los mensajes o llamadas fuera de la jornada laboral suele venir acompañado de una cláusula que también resta eficacia al derecho y que, sin embargo, debe considerarse legítima, máxime cuando la contempla el propio legislador estatal. Queriendo significar lo mismo, son diversas las coletillas empleadas

\footnotetext{
39 Medida debidamente criticada por la doctrina, puesto que no obliga a los superiores jerárquicos a "predicar con el ejemplo", puesto que les permite seguir enviando correos a sus subordinados fuera de su jornada laboral. Cfr. MIÑARRO YANINI, Margarita, "La desconexión digital...”, op. cit., p. 14.

${ }^{40}$ Por todos, MIÑARRO YANINI, Margarita, "La desconexión digital...”, op. cit., pp. 17-18.

${ }^{41}$ Una regulación como ésta "resta más que suma", como acertadamente concluye MIÑARRO YANINI, Margarita, "La desconexión digital...", op. cit., p. 17.
} 
para señalar que el derecho puede vulnerarse o reducirse ante "causa de fuerza mayor" $(7,42,54,62)$ o "circunstancias de urgencia" $(25,37)$ o "excepcionales" (1, 47 y 65) ligadas a necesidades de prestación de servicios a clientes (59), debiendo valorarse "las diferentes casuísticas y tratamientos diferenciados que puedan existir" (6, 43 y 64).

En concreto, según se indica en los convenios de empresas vinculadas a Telefónica (42 y 54): "No serán de aplicación las medidas que garantizan el derecho a la desconexión digital en los casos en que concurran circunstancias de causa de fuerza mayor o que supongan un grave, inminente o evidente perjuicio empresarial o del negocio, cuya urgencia temporal necesita indubitadamente de una respuesta inmediata". Conviene alertar aquí acerca del limitado margen de interpretación que, en mi opinión, cabría darle al referido "perjuicio empresarial o del negocio" en aras a proteger el derecho que debe disfrutar el trabajador por mandato legal.

Los citados convenios precisan tres matices: por un lado, que en tales casos las empresas contactarán con el trabajador preferiblemente por teléfono para justificarle la situación de urgencia; por otro lado, que "el tiempo de trabajo así requerido podría calificarse como hora extraordinaria, de conformidad con lo establecido en cuanto a jornada y/o política de horas extraordinarias en el marco laboral vigente en cada momento", lo cual podría venir inspirado en el convenio colectivo de BMW Group de 2014; en último lugar, se recuerda que "aquellas personas trabajadoras que permanezcan a disposición de la Compañía y perciban, por ello, un complemento de «disponibilidad» u otro de similar naturaleza" quedan excluidas del derecho a no responder los correos o llamadas. En similares términos, el convenio colectivo del consorcio de servicios de La Palma (70) y los convenios colectivos relativos a Orange Espagne (37 y 38) también excusan la vulneración del derecho a la desconexión digital ante circunstancias que puedan requerir una respuesta inmediata para evitar un "posible perjuicio empresarial" y matizan que tal derecho no se reconoce a quienes se encuentren "en situación de guardia, disponibilidad, reten, o supuestos similares".

En otro orden de cosas, el artículo 6 del convenio colectivo de la empresa Centro Farmacéutico SL (25) merece una doble distinción. En primer lugar, si bien inicialmente advierte de la posibilidad de enviar correos electrónicos o realizar llamadas telefónicas en casos de urgencia, seguidamente aclara que "aun así, el empleado tendrá la facultad de poder atender o no, cualquier tipo de comunicación".

En segundo lugar, ese precepto es el único de entre todos los analizados que declara el compromiso a establecer "anualmente" jornadas de formación para la sensibilización del personal sobre un uso razonable de las herramientas tecnológicas que evite el riesgo de fatiga informática (25). Es decir, aunque son muchos los que, haciéndose eco de la norma legal, apuntan a la formación como medida a tener en cuenta para implementar el derecho, incidiendo en la autodisciplina (30, 49 y 53) esta empresa es la única que se obliga a proporcionar cada año jornadas de formación y sensibilización a sus trabajadores -por supuesto, no necesariamente los mismos todos los años- sobre dicha temática. En fin, como se ha comentado anteriormente, otros acuerdos colectivos han destacado asimismo 
la importancia de la formación específica en gestión del tiempo de trabajo y la formación continua para trabajadores y delegados de prevención (14).

De cara a diseñar e implementar los modos de ejercicio del derecho a la desconexión digital algún convenio se pronuncia a favor de "implantar los correspondientes procedimientos de consulta, participación y negociación que regulen las modalidades de ejercicio del derecho a la desconexión digital" (18) y elaborar una guía de buenas prácticas (24), como también es relativamente frecuente la delegación de la tarea en la comisión de trabajo de asuntos sociales -cuya propuesta, sin embargo, no será vinculante para la empresa- (8) o en una comisión creada ad hoc por las empresas con convenio propio (51 y 42) para tratar los temas vinculados a la digitalización. Las funciones de vigilancia y resolución de conflictos en torno a tal implementación se reservan a la comisión paritaria del convenio $(5,61,32$ y 66). Incluso resulta plausible la original medida prevista en el II Plan de Igualdad de Caixabank (58), relativa a la creación de un circuito interno de incidencias, debiéndose resolver éstas en un plazo máximo de 7 días por la comisión de seguimiento.

En fin, lo que resulta evidente es que los dispositivos digitales no pueden ser motivo para la extensión de la jornada laboral, ni para la interrupción de los tiempos de descanso o licencias (3), por lo que, en virtud de la garantía de indemnidad y aunque no era necesario recordarlo ${ }^{42}$, "las empresas no podrán tomar medidas sancionadoras contra las personas trabajadoras que hagan uso efectivo de su derecho a la desconexión digital implantado en el seno de la empresa" (51 y 64), de modo que "no repercutirá negativamente en el desarrollo profesional de las personas trabajadoras" (42 y 54).

\section{CONCLUSIONES.}

Asumiendo la hiperconectividad digital como un factor de riesgo psicosocial, causante de estrés y otros riesgos psicosociales, estaremos en disposición de actuar de forma preventiva sobre el problema que nos traen los actuales modos de vida, tan digitalizados $\mathrm{y}$ al alcance de $\operatorname{todos}^{43}$.

El reconocimiento de un derecho siempre debe asumirse como una buena noticia, no siendo el derecho a la desconexión digital una excepción, a pesar de las numerosas críticas que ha recibido. No les falta razón a estas voces críticas, puesto que, a fin de limitar una eventual exigencia empresarial de conectividad permanente ${ }^{44}$, así como para proteger al sujeto de los riesgos que se pudiesen derivar de tan prolongada exposición a dispositivos tecnológicos, el ordenamiento jurídico español era ya suficientemente completo.

\footnotetext{
${ }^{42}$ MIÑARRO YANINI, Margarita, "La desconexión digital...”, op. cit., pp. 14 y 18.

${ }^{43}$ En este sentido se pronunció "tempranamente" el Tribunal Constitucional en su sentencia núm. 160/2007, de 2 de junio, recordando que el derecho a la protección de la integridad psíquica (y, cabría añadir, de la integridad física) exige actuar antes de que se produzca el daño.

${ }^{44}$ Sentencia de la Audiencia Nacional, de 17 de julio de 1997, que declara la nulidad de las instrucciones empresariales que reclamaban una conectividad permanente de los trabajadores a los teléfonos móviles.
} 
En cambio, el nuevo derecho reconocido tiene varias virtudes, a mi parecer. Por un lado, cumple una función indiscutible, cual es la función pedagógica de los límites y ejercicio de los derechos al descanso y a la conciliación laboral, familiar y personal, máxime ante una cuestión que, sin ser novedosa, sí preocupa cada vez más; esto es, el influjo de las tecnologías en las condiciones laborales.

Por lo que respecta a la redacción de la norma española, el uso de determinados condicionales y ciertas declaraciones programáticas en torno al derecho al descanso parecen menos garantistas para el trabajador que la regulación ya existente anteriormente sobre ordenación del tiempo de trabajo. Pero, en tales excepcionales casos, las normas de derecho necesario, como son en su mayoría las que ordenan el tiempo de trabajo, no se van a ver afectadas por unas nuevas normas que dispongan tal regulación en instituciones de igual o menor rango, por aplicación de los principios laborales más básicos.

Como se ha comprobado, muchos acuerdos colectivos reducen el derecho a la desconexión digital a la libertad del trabajador para ignorar $^{45}$ los mensajes recibidos. En mi opinión, creo que es necesario incidir sobre la cualidad de la desconexión como obligación empresarial, por los motivos que se han expresado en las páginas anteriores.

Desde el punto de vista de la seguridad y salud del trabajador, al enumerarse los principios de la acción preventiva, el artículo 15 de la LPRL indica que la finalidad principal de toda acción preventiva ha de ser evitar el riesgo, lo cual pasa por evitar que los mensajes lleguen a alterar el tiempo de descanso del trabajador.

Si nos centramos en la cuestión de la mensajería o correo, atendiendo al principio de "combatir los riesgos en su origen" actuar sobre el foco implicaría prohibir terminantemente al empleador enviar un mensaje a sus empleados fuera de la jornada de trabajo $^{46}$. Sin embargo, desde mi punto de vista este objetivo puede cumplirse de una manera más beneficiosa para ambas partes. Se trata de hacer buen uso de los avances tecnológicos, atendiendo así a otro principio preventivo, cual es el de "tener en cuenta la evolución de la técnica". Así pues, concibiendo al empresario como un sujeto vulnerable más y sabiendo que es preferible "adoptar medidas que antepongan la protección colectiva a la individual", no es preciso impedir al empleador enviar mensajes al trabajador una vez que ha terminado su jornada laboral, ya que tal restricción también podría afectar negativamente sobre la salud laboral del empleador, en tanto que no podrá descansar completamente sabiendo que tiene un mensaje pendiente de redactar y/o enviar.

\footnotetext{
${ }^{45}$ En la segunda acepción prevista por la Real Academia de la Lengua Española, consistente en "no hacer caso de algo o de alguien, o tratarlos como si merecieran atención”. Diccionario de la Real Academia de la Lengua Española, Ignorar, disponible en https://dle.rae.es/?w=ignorar (última consulta: 29.10.2019).

${ }^{46}$ Por todos, TALÉNS VISCONTI, Eduardo Enrique, "La desconexión digital en el ámbito laboral: un deber empresarial y una oportunidad de cambio para la negociación colectiva”, Información Laboral, núm. 4, 2018, p. 207. En el mismo sentido, la "NTP1123: Las tecnologías de la Información y la Comunicación (TIC) (II): factores de riesgo psicosocial asociados a las nuevas formas de organización del trabajo", de 2018, indica a modo de ejemplo de política de desconexión digital la instalación de "sistemas restrictivos de desconexión automática que inhabilite la recepción y el envío de correos electrónicos fuera de la jornada laboral para evitar una "jornada laboral sin fin"”. También resultan reiterativas en dicho texto las propuestas preventivas de organizar las consabidas (por la experiencia de Google o Intel) "siestas digitales", establecer días sin correo electrónico o evitar la confusión de las cuentas de correo corporativas y personales.
} 
Existen al efecto suficientes soluciones informáticas y digitales ${ }^{47}$, entre ellas la posibilidad de indicar la hora exacta a la que se desea que el sistema envíe la notificación.

Ahora bien, la obligación de abstenerse en el uso de medios digitales o electrónicos alcanza también al trabajador ${ }^{48}$, aunque tendrá un efecto de corte más moral que jurídico, a priori. No obstante, el régimen administrativo sancionador recaerá principalmente sobre el empresario y, excepcionalmente, sobre el trabajador ${ }^{49}$, puesto que sería contraproducente e injusto sancionar a este último cuando cabría presumir iuris tantum ${ }^{50}$ que su actitud favorable a la hiperconectividad viene provocada de manera indirecta, y quizás disimulada, por exigencias de su empleador ${ }^{51}$. Efectivamente, la realidad demuestra que a menudo el trabajador, consciente o inconscientemente, por razones de miedo al despido, a caer en desgracia, o por empatía con el cliente, ofrece una disponibilidad que rebasa los límites de su propia dignidad humana.

Por último, considero que una eventual modificación del precepto que regula el derecho no debería entrar a determinar el nivel de negociación donde debe concretarse el mismo, porque tanto la negociación de ámbito empresarial como la de ámbito sectorial se pueden beneficiar mutuamente y, ante conflicto entre ellas, habrá que estar a la materia discutida, no siendo necesario preestablecer un orden de preferencia entre una y otra. Sobre todo, cuando en este punto cabe destacar tanto la idoneidad del convenio de empresa para recoger las particulares características digitales de las relaciones laborales existentes en la misma, como las virtudes del convenio sectorial a la hora de preestablecer un marco de referencia básico en materia de desconexión digital ${ }^{52}$.

Con todo, aunque la revisión de los pactos colectivos refleja que los negociadores continúan aportando más sombras -reflejadas en forma de reiteración, buenas intenciones, ambigüedad o, incluso, reducción contra legem del derecho a la desconexión digital- que luces, algunos de los últimos acuerdos, como por ejemplo los relativos al sector comercio de Valladolid y Segovia, así como los planes de igualdad firmados por algunas entidades

\footnotetext{
${ }^{47}$ Como apunta ROTA, Anna, op. cit., p. 292, cita 2, Deutsche Telekom cuenta desde hace años con unas aplicaciones sofisticadas preparadas para seleccionar los mensajes en base a su urgencia. Se trata, de hecho, de una medida explícitamente prevista en el reciente Plan de igualdad de Caixabank (58): "Se utilizará el envío retardado de correos electrónicos". En el mismo sentido, MOLINA NAVARRETE, Cristóbal, op. cit., 2017, p. 281.

${ }^{48}$ Como sugería el Acuerdo sobre la duración del tiempo de trabajo del Grupo Réunica, de 29 de enero de 2014. En sentido opuesto, puede verse el art. 65 del Convenio colectivo de CTC Externalización, SLU (71). 49 Aunque un sector doctrinal ha sugerido una tipificación específica, tales infracciones y sanciones se podrían extraer de las que ya se encuentran tipificadas en la LISOS. Véanse, en especial, los artículos 7.5, 7.10, 8.11, 11 y siguientes, 39.3 y 40.2 de la LISOS, relativos a las infracciones del derecho al descanso y en materia de prevención de riesgos laborales, respectivamente. Cfr. PURCALLA BONILLA, Miguel Ángel, op. cit., p. 109.

50 Sin perjuicio, pues, de que el empresario pueda demostrar que ha ejercido su función preventiva in vigilando y, en su caso, su facultad disciplinaria a modo disuasorio.

${ }^{51}$ Con todo, algunos convenios colectivos insisten en reconocer y formalizar el derecho a la desconexión digital "como un derecho, aunque no como una obligación" (42 y 54, relativos a empresas vinculadas a Telefónica), lo cual ha recibido la debida crítica por parte de MIÑARRO YANINI, Margarita, "La desconexión digital...", op. cit., p. 15.

52 Se coincide, en este punto, con TERRADILLOS ORMAETXEA, Miren Edurne, "El derecho a la desconexión...", op. cit., p. 21.
} 
bancarias, marcan algunas de las sendas a seguir y permiten mantener un halo de esperanza.

\section{BIBLIOGRAFÍA}

AGUILERA IZQUIERDO, R. y CRISTÓBAL RONCERO, R., "Nuevas tecnologías y tiempo de trabajo: el derecho a la desconexión tecnológica", en AA.VV., El futuro del trabajo que queremos, Vol. 2, Madrid, 2017.

ALEMÁN PÁEZ, Francisco, "El derecho a la desconexión digital. Una aproximación conceptual, crítica y contextualizadora al hilo de la "Loi Travail No 2016-1088"”, Trabajo y Derecho, núm. 30, 2017.

BARRIOS BAUDOR, Guillermo L., "El derecho a la desconexión digital en el ámbito laboral español: primeras aproximaciones”, Aranzadi Doctrinal, núm. 1, 2019.

BAUMAN, Zygmunt, Modernidad líquida, Fondo de cultura económica, México, 2003.

BAYLOS GRAU, ello debería haberse efectuado en el artículo 4 ET. Vid. "La garantía de los derechos digitales en el trabajo. El proyecto de Ley ya aprobado en el Congreso. Un texto decepcionante", en su blog Según Antonio Baylos..., disponible en https://baylos.blogspot.com/2018/11/la-garantia-de-los-derechos-digitales.html (última consulta: 29.10.2019).

GONZÁLEZ COBALEDA, Estefanía, "Riesgos psicosociales, derechos fundamentales y NTIC: una perspectiva de protección diferente”, en CEF-RTSS Comentarios y Casos Prácticos, núm. 387, 2015.

IGARTUA MIRÓ, María Teresa, "El derecho a la desconexión digital en la Ley Orgánica $3 / 2018$, de 5 de diciembre, de protección de datos personales y garantía de los derechos digitales: más forma que fondo en la configuración del derecho", CEF-RTSS, núm. 432, 2019.

KARASEK, Robert, "Job demands, job decision latitude, and mental strain: implications for job redesign", Administrative Science Quarterly, no. 24, 1979.

LAFARGUE, Paul, El derecho a la pereza, Mandala, Madrid, 2017.

MATHIEU-GÉNIAUT, Chantal et al, "Le droit à la déconnexion: une chimère?", Revue de droit du travail, no. 10, 2016. 
METTLING, Bruno, "Informe Mettling, para adaptar el trabajo a la transformación digital”, Ministerio de Trabajo, Migraciones y Seguridad Social: Actualidad Internacional Sociolaboral, núm. 194, 2015.

MIÑARRO YANINI, Margarita, "La desconexión digital en la práctica negocial: más forma que fondo en la configuración del derecho", CEF-RTSS, núm. 440, 2019.

MOLINA NAVARRETE, Cristóbal, "Jornada laboral y tecnologías de la infocomunicación: "Desconexión digital", garantía del derecho al descanso", Temas Laborales, núm. 138, 2017.

NAVAL, Concepción et al, "Sobre la necesidad de desconectar: algunos datos y propuestas", Education in the Knowledge Society, vol. 17, n. 2, 2016.

PALOMEQUE LÓPEZ, Manuel Carlos, Los derechos laborales en la Constitución Española, Centro de Estudios Constitucionales, Madrid, 1991.

PURCALLA BONILLA, Miguel Ángel, "Control tecnológico de la prestación laboral y derecho a la desconexión: claves teóricas y práctica”, Anuario IET de trabajo y relaciones laborales, Vol. 5, 2018.

REQUENA MONTES, Óscar, Los derechos individuales de formación en el artículo 23 del Estatuto de los Trabajadores, Tirant lo Blanch, Valencia, 2019.

RODRÍGUEZ-PIÑERO ROYO, Miguel, en el turno de preguntas de la mesa sobre "Desconexión digital", del Congreso sobre Automatización, vigilancia y control de trabajadores y derechos fundamentales, Valencia, 12 de septiembre de 2019. Disponible en https://www.youtube.com/watch?time_continue=89\&v=nF1QNYyPe3c (última consulta: 29.10.2019).

ROTA, Anna, "El debate europeo sobre el derecho a la desconexión en las relaciones de trabajo", en AA.VV., Anuario internacional sobre prevención de Riesgos Psicosociales y calidad de vida en el trabajo 2016: Nuevas tecnologías de la Información y la Comunicación y Nuevos Riesgos en el Trabajo, UGT, 2016.

TALÉNS VISCONTI, Eduardo Enrique, "La desconexión digital en el ámbito laboral: un deber empresarial y una oportunidad de cambio para la negociación colectiva", Información Laboral, núm. 4, 2018.

TERRADILLOS ORMAETXEA, Miren Edurne, "El derecho a la desconexión digital en la ley y en la incipiente negociación colectiva española: la importancia de su regulación jurídica", Lan Harremanak, 2019, 42, In press. Disponible en 
VALLECILLO GÁMEZ, María Rosa, "El derecho a la desconexión: ¿"Novedad digital” o esnobismo del "viejo" derecho al descanso?", CEF-RTSS, núm. 408, 2017.

\section{ANEXO. LISTADO DE CONVENIOS COLECTIVOS Y PLANES DE IGUALDAD CON REFERENCIA EXPRESA A LA DESCONEXIÓN DIGITAL}

\begin{tabular}{|c|c|}
\hline № & FECHA Y LUGAR PUBLICACIÓN \\
\hline & $20170921 \mathrm{BOE}$ \\
\hline & 20180104 \\
\hline & $20180118 \mathrm{BOE}$ \\
\hline & 20180418 BOP Barcelona \\
\hline & 20180601 No publicado \\
\hline & 20180907 DO Gen. Cataluña \\
\hline & $20181208 \mathrm{BOE}$ \\
\hline & $20190110 \mathrm{BOE}$ \\
\hline & $20190126 \mathrm{BOE}$ \\
\hline 10 & $20190204 \mathrm{BOE}$ \\
\hline 11 & 20190214 BO Com. de Madrid \\
\hline 12 & 20190214 BOP Alicante \\
\hline 13 & 20190319 BOP Huelva \\
\hline 14 & 20190416 BO Navarra \\
\hline 15 & 20190516 BOP Huelva \\
\hline 16 & 20190517 BOP Burgos \\
\hline 17 & 20190613 BOP Sevilla \\
\hline 18 & 20190708 BO Aragón \\
\hline 19 & 20190709 BOE \\
\hline 20 & $20190709 \mathrm{BOE}$ \\
\hline 21 & $20190716 \mathrm{BOE}$ \\
\hline 22 & $20190716 \mathrm{BOE}$ \\
\hline 23 & 20190722 BO Com. de Madrid \\
\hline 24 & $20190722 \mathrm{BOE}$ \\
\hline 25 & $20190731 \mathrm{BOE}$ \\
\hline 26 & $20190819 \mathrm{BOE}$ \\
\hline 27 & $20190820 \mathrm{BOE}$ \\
\hline 28 & 20190821 BOP Sevilla \\
\hline 29 & 20190822 BO Cantabria \\
\hline 30 & 20190828 BOP Alicante \\
\hline 31 & 20190903 BO Navarra \\
\hline 32 & 20190903 BOP Alicante \\
\hline 33 & 20190916 BOP Barcelona \\
\hline 34 & 20190925 No publicado \\
\hline 35 & 20191002 BOE \\
\hline 36 & 20191003 BOP Girona \\
\hline 37 & $20191018 \mathrm{BOE}$ \\
\hline 38 & $320191018 \mathrm{BOE}$ \\
\hline 39 & 20191022 BOP Barcelona \\
\hline 40 & 20191026 BO Com. de Madrid \\
\hline 41 & 20191104 BOP Huelva \\
\hline 42 & $20191113 \mathrm{BOE}$ \\
\hline 43 & 20191118 BOP Barcelona \\
\hline 44 & 20191123 BO Com. de Madrid \\
\hline 45 & 20191123 BO Com. de Madrid \\
\hline 46 & 20191123 BO Com. de Madrid \\
\hline 47 & $20191203 \mathrm{BOE}$ \\
\hline 48 & 30191205 BOE \\
\hline 49 & 20191210 BOP Alicante \\
\hline 50 & 20191213 BO Com. de Madrid \\
\hline 51 & $20191219 \mathrm{BOE}$ \\
\hline 52 & 20191221 BO Com. de Madrid \\
\hline 53 & 20191228 BO Com. de Madrid \\
\hline 54 & $20200107 \mathrm{BOE}$ \\
\hline 55 & 20200114 BO Navarra \\
\hline 56 & 20200121 BOP Jaén \\
\hline 57 & 20200122 BOP Barcelona \\
\hline 58 & 20200124 En prensa y web \\
\hline 59 & $20200130 \mathrm{BOE}$ \\
\hline 60 & 20200131 BOP Pontevedra \\
\hline 61 & $20200204 \mathrm{BOE}$ \\
\hline 62 & 20200205 BOP Valladolid \\
\hline 63 & 20200214 DO Gen. De Cat \\
\hline 64 & 20200217 BOP Segovia \\
\hline 65 & $20200227 \mathrm{BOE}$ \\
\hline 66 & $20200228 \mathrm{BOE}$ \\
\hline 67 & 20200302 BO TH Álava \\
\hline 68 & $320200311 \mathrm{BOE}$ \\
\hline 69 & $20200311 \mathrm{BOE}$ \\
\hline 70 & 20200313 BOP S.C \\
\hline 71 & $20200318 \mathrm{BO}$ \\
\hline
\end{tabular}

CC Grupo Axa

IX Acuerdo Marco del Grupo Repsol

XVI CC de la ONCE y su personal

CC de l'empresa Barcelona Cicle de l'Aigua, SA (BCASA)

Acuerdo para la distribución de la jornada en Ikea ibérica SA

Acuerdo Interprofesional de Cataluña

CC Empresa Philips Ibérica, S.A.U

III CC de la empresa Activa Innovación y Servicios, S.A.U.

Plan de Igualdad de la empresa Hipermercados Carrefour (Centros Comerciales Carrefour,

S.A., Carrefour Norte, S.L. y Carrefour Navarra, S.L.)

II Plan de Igualdad del Grupo de Empresas Champion (Supermercados Champion, S.A. y Grupo Supeco Maxor, S.L.)

CC del sector de Industria, Servicios e Instalaciones del Metal de la Comunidad de Madrid CC Institución ferial alicantina

CC del personal laboral de la comarca de la Jacetania

Revisión del acuerdo intersectorial de Navarra sobre Relaciones Laborales

CC Empresa Autoamtax sociedad cooperativa andaluza

CC de ámbito provincial para la actividad de oficinas y despachos

CC Empresa EUI IT Global Services A.I.E.

II CC Grupo Aramón

CC Industria del calzado

II CC de la Asociación para la Gestión de la Integración Social

II CC de las entidades públicas empresariales Administrador de Infraestructuras Ferroviarias y Administrador de Infraestructuras Ferroviarias de Alta Velocidad

II CC del Grupo Acciona Energía

CC del comercio del metal

II CC de oficinas corporativas del Grupo Siemens Gamesa

CC Centro Farmacéutico SL

III CC de Cash Converters SL y Sociedades Vinculadas

CC Estatal de perfumería y afines

CC Empresa EUI Limited Sucursal

CC para el sector de abogados, procuradores y graduados sociales de Cantabria

II Plan de Igualdad de Torrevieja Salud

CC de la empresa Unicarriers Manufacturing Spain SA

CC de Editorial Prensa Alicantina

$\mathrm{CC}$ del sector de majoristes de fruites, verdures, hortalisses, plàtans i patates de la província de Barcelona

Acuerdo del BBVA sobre registro de jornada y desconexión digital

CC estatal para las industrias del curtido, correas y cueros industriales y curtición de pieles para peletería

CC del sector del comerç en general de la província de Girona

$\mathrm{CC}$ de Orange Espagne SAU

CC de Orange España Comunicaciones Fijas, SLU

CC de comercio e importadores de artículos fotográficos, vídeo y sonido

$\mathrm{CC}$ del sector de comercio vario

CC de la empresa Ambulancias La Cinta SL

II CC de empresas vinculadas para Telefónica de España, SAU, Telefónica Móviles España, SAU y Telefónica Soluciones de Informática y Comunicaciones de España, SAU $\mathrm{CC}$ del comercio textil

CC de la empresa Sociedad Española de Fútbol Profesional

CC de la empresa Servicios, Infraestructuras y Mantenimiento en el Deporte, S.L.U.

CC de La Liga

CC del Grupo Santander

CC de la Liga Nacional de Fútbol Profesional

II Plan de Igualdad de Vinalopó Salud

CC de la Red Universal de Marketing y Bookings Online SA Rumbo

CC estatal de la Industria, tecnología y servicios del sector del metal

CC estatal de la Industria, tecnologit Retail Group Madrid

CC de Renault Retail G

CC de Telefónica Ingeniería de Seguridad

Plan de Igualdad Unicarriers Manufacturing Spain

CC de Siro Jaén SLU

CC de l'empresa Fundació de Gestió Sanitària de l'Hospital de la

Santa Creu i Sant Pau

II Plan de Igualdad de Caixabank

CC de Grupo Selecta

Plan de igualdad J.L. Froiz SL

CC de Servicios Dix 2012

$\mathrm{CC}$ del sector comercio

$\mathrm{CC}$ delsector d'oficines idespatxos de Cataluna

$\mathrm{CC}$ del sector comercio

CC de Carlson Wagonlit España, SLU

CC de Siemens Healthcare, SLU

CC para la empresa Fundación Estadio Vital Fundazioa

CC estatal de estaciones de servicio

CC de BT España, Compañía de servicios globales de telecomunicaciones, SAU

CC del Consorcio de servicios de La Palma

CC de CTC Externalización, SLU
"DESCONEXIÓN DIGITAL"

Art. 14

Art. 16.9

Anexo 3 Apartado B5

Art. 65

Punto 13

Capítulo XV

Art. 46

DA 1a

Objetivo concreto 5.2

Objetivo concreto 5.2

Art. 51.Ter

Art. 26

DA 10

Punto 8

Art. 26

Art. 31

Art. 16.10

DA 2a

DA 2

EEMM y Art. 78

Cláusulas 13 y 15

Art. 84 bis

Art. 21

Art. 47

Art. 6

DA 3 a

Art. 16

Art. 16.10

Art. 42

Objetivo 1.4

DF 13 y Anexo IX

Art. 8

Art. 42

Art. 85

Art. 85

Art. 52

Art. 23.4

Art. 23.4

Art. 76

Art. 89

Art. 89

Anexo XIII

DA 8

Art. 32

Art. 32

Art. 32

Art. 11

Art. 32

Objetivo 1.4

Art. 19.5

Arts. 48 y 120

Art. 6

Apartado IV.7

Art. 41 y Anexo

Punto 10

Apartado IV.4

Art. 90

Art. 44

Apartado 6.3

Art. 20

Art. 54

Art. 38

Art. 78

Art. 24

Art. 43

Anexo 5

Capítulo XIV

Art. 19

Art. 65 